\title{
II. \\ Über die Bedeutung der gegenwärtigen Zeit als Wendepunkt in der Geschichte der Menschheit. Von \\ Rudolf Tönnis, Berlin-Dortmund.
}

Die weltgeschichtlichen Ereignisse unserer Zeit zwingen alles Denken in ihren Bann, lenken den Blick in verstärktem Maße auf die Tatsächlichkeiten des Lebens und rufen eine große passive Einheit der verschiedensten Denkrichtungen hervor. Eine notwendige Wirkung, die uns auf die Lehren, die uns das Leben jetzt gibt, vorbereitet und dafür empfänglich macht.

Wenn ein gewaltiges Unwetter über eine Gegend dahinbraust und allem, was lebt, mit Vernichtung droht, da vertragen sich Wolf und Schaf. Die Fragen ider Selbsterhaltung, die jeden mit gleicher Wichtigkeit angehen, sind die 'nächstliegenden, und aller Wille richtet sich in gleichem Sinne gegen den gemeinsamen Feind. Unter der Wucht der g e me in same n Interessen treten alle unterschiedlichen Meinungen in den Hintergrund.

Gegeniiber dem früheren Parteihader auf allen Gebieten wirkt die gegenwärtige Volkseinigkeit in ihrer erhebenden Größe wie eine wunderbare Erlösung von allen „Engherzigkeiten und Kleinlichkeiten", die das wissenschaftliche und öffentliche Leben scheinbar ganz unnötig zu zersplittern schienen. Nun ist plötzlich ein gemeinsames, alle vereinendes Ziel da; e in beherrschender Gedanke bewegt das ganze Volk.

Aber der gemeinsame Gedanke, so groß und qualitativ wertvoll er auch sein mag, ist mehr ein äußerlicher, d. h. ein durch äußere Anlässe hervorgerufener. Er trägt zwar wesentlich zur Klärung vieler Streitfragen bei und hat seine bleibende Rückwirkung auf alle 
inneren Verhältnisse, wie wir schon jetzt sehen und wie das in der Geschichte nach allen großen Kriegen der Fall war, aber die Lösung grundsätzlicher Gegensätze trägt er nicht in sich, trägt er wenigstens zunächst nicht in sich.

Von allen großen Ereignissen werden große Wirkungen erwartet. Wer aber die Hoffnung hegt, daß mit der äußeren Einigkeit auch die Zwiespältigkeit der innerpolitischen Gesinnungen, die ja die weitesten Kreise berühren, beigelegt sein könnte, wird sich schwer enttäuscht sehen. Den Wandel der Gesinnung èrwartet jede Partei von der andern; sie selbst weiß nichts von ihrem eigenen bösen Geist der Unverträglichkeit.

Die alten. Streitfragen sind nur eine Zeit lang von der Tagesordnung abgesetzt und ad acta gelegt. Auch sorgt die Zensur für Orientierung der $z u$ erörterndien Fragen an der Zeitlage. Sie können weder durch ein großes Zeitereignis erledigt, noch auf andere Weise beseitigt werden. Es sind eben innerpolitische Le.bensaufgaben, die gelöst werden müssen, es gibt keinen andern Weg.

Die innerpolitischen Fragen und solche anderer Lebensgebiete treten nur solange in den Hintergrund; wie die einende Ursache anhält und stark genug anhält. Ist das Kriegsgewitter vorbei, so müssen auch notwendigerweise die alten Fragen wieder auftauchen und der alte Hader und Streit um grundsätzliche Meinungsverschiedenheiten, (die letzten Endes in einer uneinheitlichen Lebensauffassung ihre gemeinsame Wurzel haben), ist wieder da.

Unbemerkt wird vielfach unsere gegenwärtige Einigkeit von denjenigen; die hinter den Ereignissen eine tiefere Wandlung der gesamten Menschheit vermuten, in uniklarem Verständnis des Drängens des deutschen Zeitgeistes in glattem Hinüberfluß auf diese übertragen und von der Zukunft erwartet. Die Ereignisse der ersten Kriegsmonate und die beispiellosen ferneren Erfolge unserer Heere, schienen ja die kühnsten Erwartungen $z u$ rechtfertigen. Im Vierlaufe fast $z$ weier Kriegsjahre ist jedoch eine erheblich klarere Erkenntnis der Sachlage eingetreten, die zu nüchternster Betrachtung derselben zwingt und die Hoffnungen der Zukunft auf ein durch die Tatsachen berechtigtes Maß herabsetzt. Die Meinungen über höhere Ziele sind daher recht verschieden. 
Während der eine jede Erörterung nicht realistischer Ideen von der Hand weist und alle über die nächstliegenden praktischen Forderungen des Lebens hinausgehende Fragen für verfehlt wähnt, hält der andere in der Stille an unbestimmten fernen Zielen fest: hinter dem Dunkel ides Gestehens verbirgt sich mehr, als nur p o li t is che oder wirtschaftliche Meinungsverschiedenheiten. Um Meinungsverschiedenheiten handelt es sich allerdings, aber sie liegen tiefer, sie gehen -aus von grundsätzlich verschiedenen Weit- und Lebensauffassungen. Es kämpft der sachlich-idealistische deutsche Geist gegen den naturhaft-egoistischen englischen!

Wenn auch vom dleutschen Geist viel zu erhoffen ist, so ist er doch keineswegs heute soweit gereift, daß eine durchschlagende Wirkung auf die Lebensauffassung fremider Nationen schon jetzt $\mathrm{zu}$ erwarten wäre. Diese Gedanken sind' es auch keineswegs, die uns einen; wenigstens walten sie nicht bei der großen Mehrheit vor. Die das Volk beherrschende Einigkeit müssen wir zunächst trennen von denjenigen, die wir eventuell für die Zukunft der Menschheit durch den deutschen Idealismus erwarten.

Der Krieg, d. h. das Bewußtsein, daß uns ein schweres Unrecht-geschieht, sowie der Zorn gegen die gemeinsamen Feinde, sind die Ursache der Einigkeit im allgemeinen. Der uns gegenwärtig beseelende Einheitsgedlanke ist nur spontan unter dem Zwang der Notwendigkeit entstanden, erstreckt sich auch nur auf unsere politis che Lebenseinheit und ist wegen seines subjektiven Charakters nicht geeignet, in Beziehung zur Menschheit zu stehen. Diese wird gerade durch subjektive Affekte und nationale Meinungen zerrissen. So erbittert wie heute haben die Nationen in ihren Gegensätzen einander noch nie gegenübergestandien. Es ist, als ob die Harmonie der Menschheit in der Tat ein leerer Traum sei.

Aber trotzdem: hinter der Ursache der deutschen Einheit, hinter dem Bewußtsein des schweren Unrechts wird unbewußt mehr .e $\mathrm{mpfunde} n$ als bewußt erkannt wird, und wenn auch zwischen dem deutschen Einheitsbewußtsein und der Harmonie der Menschheit eine zewaltige Kluft liegt, eine nähere Beziehung ist $\mathrm{doch}$ vorhandien. Es fragt sich nämlich, ob nicht das Rechtsbewußtsein, das in uns lebt, sich auf . Tatsächlichkeiten stützt, die dem Recht an sich, die dem Recht als solchem also von Haus aus $\mathrm{zu}$ Grundie -liegen, $d . h$. aber ein solches wäre, das nicht nur uns er m nationalen 
Rechtshewußtsein entsprächc, sondern dem aller Völker, wenn sie zur Anerkentnung dieser ihrem Standpunkt übergeordneten Wahrheit gebracht werden könnten.

Das müßte eine Wahrheit sein, die nicht von unserem persönlich nationalen Willen abhängig wäre, sondern a n sich bestände, also dem Leben als solchem zu Grunde läge und dem sich jeder als letzte Wahrheit zu fügen hätte. Freilich gibt es diese Wahrheit heute noch nicht, sie läßt sich aber gewinnen, sie läßt sich ableiten aus der klar gesehenen Entwicklung des Lebens.

*.

Wer das Geschehen von einer höheren Warte aus betrachtet, dem muß sich die Tatsache aufdrängen, daß ja die gleichen sozialpolitischen Fragen alle Völker beschäftigen, ebenso wie die gleichen Probleme der Wissenschaft und die höchsten geistig-seelischen Lebensfragen die gesamte Menschheit betreffen. Beide Problemgruppen sind Teile eines großen geistigen Reifungsprozesses der Menschen und speziell der höheren nationalsozialen Entwicklungseinheiten derselben.

Wie aber aus dem Ausgange der kriegerischen Konflikte - in welchem Sinne'es auch sei - die Lösung der höchsten Probleme hervorgehen soll, ist zunächst ganz unklar; es ist ohne weiteres nicht $\mathrm{zu}$ ersehen, wie sich etwa die Streitfrage $z$ wischen Individualismus und Sozialismus, 'zwischen Egoismus und Altruismus, zwischen Realismus und Idealismus, zwischen Nationalismus und Universalismus, $z$ wischen Monismus und Dualismus, zwischen Wissenschaft und Religion lösen sollen; denn diese Fragen berühren sich alle und alle hängen in der Tiefe miteinanider zusammen.

Die geistige Entwicklung der Menschen ist seit der Renaisisance und Reformationszeit in wachsendem Maße eine expansive gewesen und dehnte sich schließlich so weit aus, daß die Menschen nun jede geistig-seelische Einheit und Konzentration auf a $11 \mathrm{gem}$ e in e Ideen entbehren. Eine höhere geistige Führung durch von allen anerkannte und sie in gleicher Wieise beherrschende Gedanken fehlt heute völlig.

Die geistige Zerrissenheit der Menschheit von heute ist eine wesentliche Ursache des Krieges, die bei unserer hohen Kultur als Hohn auf die Gesittung empfunden werden muß. Die alten Ideale sind verblaßt und neue sind noch nicht da. 
Einende Menschheitsgedanken barg seinerzeit das Chirstentum, aber jene Epoche war noch nicht reif, die ganze. Wahrheit $z \mathfrak{u}$ erkemnen und so verband sich mit jenem Gedankengebäude sowohl ein unwahres Weltbild als auch eine ebenso unhaltbare Lebensanschauung, d. h. soweit sie auf dogmatischer Grundlage ruht. Die christlichen Gedanken haben Kriege $z \mathfrak{u}$ keiner Zeit verhindern können, sie mußten aber im selben Maße ihre einende und verpfilichtende Kraft umsomehr einbüßen, je mehr die Erfährung allen -Einsichtsvolleren sagte, daß die Wirklichkeit des Lebens mit jenen Lehren nicht übereinstimmte. So mußte das Interesse der Völker auch in erhöhtem Maße auf nächstliegende, die eigene Zukunft betreffende Fragen zurücksinken, als daß sie sich für allgemeine Menschheitsgedanken hätten erwärmen können.

Von humanitären Menschheitsgedanken kann erst dann diese verpilichtende Kraft aüsgehen, wenn ihnen diejenigen Gedanken klar bewußt $z \mathfrak{u}$ Grundle gelegt werden können, die ihrem Wesen in der Tiefe des Unbewußten, d. h. an sich zu Grunde liegen.

Es ist nicht in Frage zu' stellen, daß die christliche Lehre hohe Wahrheitswerte in sich birgt, die alle Zeiten überdauern werden, aber ebenso richtig ist es, mit den Tatsachen zu sprechen und zu behaupten: Hätte sich die Kraft der christlichen Ideen als genügend stark erwiesen, so hätte der Krieg gar nicht entstehen können. daß er doch entstand und. Jahrzehnte hindurch unter den Augen der offiziellen Vertreter der christlichen Lehre sich vorbereiten konnte, beweist, daß die Lehre nicht aus sich heraus die Kraft besaß, das gegenwärtige Schicksal von der Menschheit abzuhalten. Sie hat das schwerste aller Dramen der Menschen nicht nur nicht von den Menschen im allgemeinen abwenden können, sondern nicht einmal von den christlichen Völkernuntere in ander. Es sind in der christlichen Lehre, noch nicht di e Wahrheiten ausgesprochen, die sich als $F$ ührer. im Leben bewähren.

Es gilt also die christlichen Ideen auf ihren wahren Wert zurückzuführen, so daß sie sich sinnvoll und ahne $Z$ wang in das an sich bestehende Lebensgetriebe einfügen. Bevor dies aber möglich ist, müssen wir das Leben selbst in seinen Grundzügen kennen und die Wahrheiten der neuen Zeit, denen jene christlichen Ideen alle in nicht gewachsen waren, $z \mathfrak{u}$ gewimen suchen. 
Einende Menschheitsgedanken können nur gewonnen werden, indem wir die Wahrheiten, die dem Leben an sich zu Grunde liegen, zu Richtlinien fürs Leben machen und aus ihnen die fehlenden Ideale ableiten.

Die bleibenden Werte, welche unsere verfließende Epoche gegenüber allen früheren Zeitperioden neu herausgebracht und zum alten Bestande des Wissens als neu hinzugefiugt hat, und die ihr den besonderen Stempel aufdrücken, konzentrieren' sich im Entwicklungsgedanken: Das Leben unterliegt in all seinen Erscheinungsformen der Entwicklung und diese Wahrheit steht jetzt ebenso im Mittelpunkt einer Weltanschauung, ist ebenso unvergänglich wie diejenige, die den ethischen Gehalt, d. h. den Kernpunkt der christlichen Lehre ausmacht. Da beide wahre, fundamental wahre Bestandteile des Lebens bilden, so können neue, allgemein anzuerkennende, einende Gedanken nur aus der Verschmelzung. beider Gedanken hervorgehen.

$*$

Wir müssen zwischen zwei christlichen Giedankenkreisen unterscheiden, die an sich nichts miteinander zu tun haben.

Der eine entspringt den in der menschlichen Natur begrundeten höheren: altruistischen Trieben und erstreckt sich auf allgemeine Menschenliebe und Betonung des Einzelmenschen wertes. Der höheren Natur entstammt auch die Sehnsucht der Mienschen nach Erlösung von dem Elend dies Daseins, nach einem höheren, lauteren, vollkommeneren Leben als dem a $11 \mathrm{~g}$ e me in en Ziel der Menschen. Diese Sehnsucht ist auch im Menschen selbst vorhanden, ist eine Eigenschaft seiner Seele, ist in seiner höheren Natur begründet und besitzt somit gleichfalls eine $\mathrm{N}$ a tur $\mathrm{gr}$ r n d $1 \mathrm{ag} \mathrm{e}$, die in allen gesitteten Völkern lebt, ob sie nun budidhistisch, mohammedanisch oder christlich sind. Aber die Erklärung, was unter diesem h ö h e r e n Leben, das uns über die Not des täglichen Lebens emporheben soll, $z \mathfrak{u}$ verstehen und der Weg, wie ein solches Leben zu erreichen ist, sowie überhaupt die $\mathrm{ErkJ}$ ä $\mathrm{r}$ u $\mathrm{g}$ des allgemeinen Weltgeschehens und des widderspruchsvollen Lebens, die sich um diese zentralen Empfindungen und Gedanken bewegt, sich also notwendigerweise mit dem inneren Sehnen verbinden muß und $\mathrm{ge}$ radezu den Inhalt der Naturgrundiage $z u$ bilden 
hat, ist bei der christlichen Lehre in wesentlichen Gesichtspunkten ebenso verfehlt, wie bei den übrigen universal-religiösen Systemen. Es. sind die willkürlich angenommenen Dogmen, sie billden den zweiten Ideenkreis.

Der unaufgeklärten Zeit ihrer Entstehung gemäß ist eine richtige Erklärung der Welt und des Lebens aber auch gar nicht zu erwarten und darum trifft jene Stifter und Verkünder keinerlei Vorwurf. Im Gegenteil, fïr ihre Zeit waren ihre oder ähnliche Lehren notwendig, die Menschheit verlangte nach Ideen, die sie befreiten, von der damals vorherrschenden Not des Daseins.

Für eine Lehre, die un s he u te n o c h befriedigen würde, waren jene Zeiten bei weitem nicht reif, sie würde, ganz abgesehen von der Unmöglichkeit ihres Entstehens, auch jenen Menschen nicht das haben nützen können, wie die ihrem Verständnis angepaßten einfachen Deutungen, die eben dem Geiste ihrer Zeit entsprungen waren.

Da die Grundlagen zu idem richtigen Weltbild noch nicht erforscht waren, so $\mathrm{mu} B$ te das alte Weltbild, sowie die Anschauing vom Leben: was seine Bedeutung und sein Sinn ist, in wesentlichen Ziigen unrichtig aușfallen.

*

Aus dem Studium der Natur hat sich im Laufe der Jahrhunderte eine solche Fülle sich gegenseitig stützenden und ergänzenden wissenschattlichen Tatsachienmaterials zusammentragen lassen, daß es sich zu dem festen Fundament einer neuen Weltanschauung ausbauen ließ.

Besonders war es der um die Mitte des vorigen Jahrhunderts iestere Gestalt gewinnende Entwicklungsgedanke, welcher Licht auf die Entstehung der Pflanzen- und Tierwelt warf, deren Existenz bis dahin allen unbegreiflich erschien, denen die naive Erklärung des mosaischen Schöpfungsberichtes nicht genügte.

Die Auslese und Anpassung im Daseinskampi bildeten Begriffe von großer Tragweite auch für das Leben des Menschengeschlechts. Nach der Entwicklungslehre steht der Mensch in enger Beziehung zum Tierreich, ist aus ihm hervorgegangen, im Gegensatz zu dier Anschauung der Kirche, die zwischen Mensch und Tier bezw. zwi- 
schen dem Menschen und der übrigen Natur eine große Klut annimmt.

Der Dascinskampf, dem die christliche Lehre aus dem Wege zu gehen suchte und der, wie jedem unbeiangenen Blick offenbar wurde, nicht allein im Pflanzen- und Tierreich, sondern auch unter den Menschen bestand, wurde plötzlich in seiner ungeheuren Bedeutung für alle Lebewesen klar. Unklar blieb jedoch seine Berechtigung oder Nichtberechtigung unter den Menschen, denn der Mensch war doch schließlich nicht mit den Tieren auf eine Stuie zu stellen, er besitzt höhere edlere Eigenschaften. Andererseits bildete der Daseinskampf, der die tüchtigen Geschöpie über die weniger tüchtigen zum Sieger machte und alle Unfähigen zurückhielt, einen wichtigen Faktor auch im Leben der Menschen; i m Kulturleben der Menschen, für welches in der christlichen Lebensanschauung gar kein rechter Platz zu finden war, denn nach den christlichen. Anschauungen war das Dichten und Trachten des menschlichen Herzens böse von Jugend auf. Diesem irdischen Dichten und Trachten entspringt aber gerade das Kulturleben mit seinen Kämpfen und seiner Not. Wer nun die Not zu bekämpfien trachtet, der darf nicht das irdische Sehnen und Wünschen, das Dichten, Trachten und Streben, das ja den ganzen Inhalt unserer Seele ausmacht, verurteilen ind den Daseinskampf verneinen und alle Zwiespältigkeit mit ihren Leiden und ihrer Not auf eine Sch uld der Menschen zurückführen wollen. Eine solch primitive Erklärung kann heute nur noch einer seichten Auffassung genügen, die von dem "wirklichen Ineinandergreifen der Lebensfaktoren keine Vorstellung besitzt. Nur in der vollen Bejahung des Lebens, in seinem Verständmis und in der sinnvollen Anpassung an die Tatsächlichkeiten desselben könnenwir eine Erklärung und langsame Lösung der Widersprüche finden.

*

Friedrich Nietzsche erfaßte zunächst die biologische Bedeutung des Entwicklungsgedankens und seine Tragweite für das Menschengeschlecht und mit heiligem Zorne wandte er sich gegen die christliche Lehre als eine Lehre erbärmlicher Sklavenmoral, die gerade die kraftvollsten und tüchtigsten Menschen unterdrïcke, dagegen alles Niedrige und Schwäcliliche geradezu künstlich großzuichite. Er predigte die alleinige Existenzberechtigung eines-Herren- 
menschentumes und die rücksichtslose Bekämpfung aller Elenden und Schwachen.

In der Befürwortuny dieses krassen Egoismus kehrt Nietzsche in gewissem Sinne wieder zu Verhältnissen zurück, wie sie zur römischen Kaiserzeit bestanden, aus jenen heraus war aber die christliche Moral entstanden; sie war eine Überwindung und Besiegung dieser egoistischen Zustände: eine Besinnung auf die eddleren Eigenschaften im Menschen, denen sie zum Siege verhalf. Damals galt der Starke alles, der Schwache nichts. Eine Rückkehr zu diesen Zuständen wäre aber kein Fortschritt.

Nietzsche überträgt mit seinen Forderungen den Daseinskampf der Tierwelt ohne weiteres auf die Menschen und läßt ihre höheren, sittlichen Eigenschaften außer acht. Diese scheinen ihm gefährlich, insofern sie die Entwicklung eines kraftvollen Menschiengeschlechts interdrücken, $d$. h. nach der $d o g \mathrm{~m}$ a t is $\mathrm{c}$ h christlichen Lehre unterdrücken. Der Hinweis, daß der Wille zur Macht nicht an sich selbst orientiert sein darf, sondern Mittel zu höheren Zwecken ist, fehlt zwar nicht bei Nietzsche. Doch wird die sittliche Disziplinierung des Machtwillens nicht genïgenid betont, denn es fehlt noch der Maßstab, woran der Wille $\mathrm{zu}$ orientieren ist.

Da die Entwicklung des Menschengeschlechts aus dem Tierreich und die Vererbbarkeit lebenstüchtiger Eigenschaften auf die Nachkommen wissenschaftlich unumstößlich feststeht, so sind mit den Lehren Nietzsches wichtige Fragen für die Zukunft des Menschengeschlechts angeschnitten, Fragen mit starkem Wahrheitshintergrunde, die nicht mehr von der Tagesordnung verschwinden können, es sei denn, daß sie gelöst werden. Die Bedeutung Nietzsches besteht demnach vorwiegend in der Stellung der neuen_Probleme, nicht in den Antworten, die er darauf gibt. Er selbst erachtet diese Fragen, die im übrigen eine ganze Reihe weiterer Probleme nach sich ziehen, auch noch nicht für grelöst.

So wie Nietzsche den Gedanken des Willens zur Macht in dem Mitteipunkt einer neuen Lehre stellt, von dem aus er alle Lebensverhältnisse betrachtet, so haben auch frühere Erzieher ider Menschheit, die die Probleme des Lebens $\mathrm{zu}$ lösen sich bemïhten, von einem bestimmten ethiṣchen Gedanken aus diese Antworten geben zu können geglaubt, die dann ebenso einfach wie einlenchtend erschienen. 
Plato zeigt den Menschen in hohen Zielen und Bildern das Ideal. Christus lehrt die Nächstenliebe, Kant verweist uns auf die Pilicht, nàch Schopenhauer kann nur das Mitleid der Ausgang aller Moral sein, Bentham stellt die Wohlfahrtsmoral als letztes Ziel auf.

So werden von diesen und anderen Denkern bestimmte Leitgedanken zu Richtlinien fürs Leben gemacht, denen sich alle Handlungen unterordnen sollen. Ein jeder hat ein anderes Hauptziel im Augse.

Die Einseitigkeit dieser Forderungen ist uns heute Lebenden, die wir allmählich einen Begriff von der Vielseitigkeit des wirklichen Lebens erhalten haben, mehr und mehr zum Verständnis gekommen.

Die Breite des Gesichtsfieldes war früheren Zeiten durch die noch fehlende Tatsachenkenntnis verengt. Solange wie die Menschen dem chaotischen Dunkel gegenüberstehen, das sich von keiner Seite recht in Angriff nehmen und erklären lassen will, ist natürlich jeder Lichtstrahl, der konsequent das Leben durchidringt, zu seiner Aufhellung wichtig. Die Philosophen konnten an Hand der Tatsachenkenntnis und gemäß ihrer individuellen Anlage zunächst nur- einseitige Richtlinien entdecken, die an sich nicht falsch, sonldern in ihrer Einseitigkeit schief gesehen sind und darum Falsches enthalten.

Wir Menschen von heute erkennen mehr und mehr, daß wir die Denker der Vergangenhieit weder ablehnen noch eines ihrer Systeme, so wie es ist, annehmen können; sie alle enthalten bleibende Werte, und die Kämpfe jener um die Wahrheit waren nicht umsonst. Wir dürfen indessen die Wirklichkeit $n$ ich $t$ bestimmte $n$ Gedanken unterordnen.

Die Wirklichkeit besteht an sich, wir müssen daher das Leben, wie es ist, erklären, d. h. aber a us ihm die Richtlinien zu gewinnen suchen, die wir brauchen. Es kann ja kein Zweifel sein, daß wir $\dot{z} u$ einer harmonischen Lebensführung weder das Ideal der Menschenliebe, noch aber die Eigenliebe, noch die Pflichterfüllung, noch das Mitleid oder die soziale Wohlfahrt usw. entbehren können. Wir bediirfen all dieser und anderer Wahrheiten, jede an ihrem Teil und jede zu ihrer Zeit. Wir bedürfen eines höchsten Gutes, das alle diese Ziele als seine Teilziele unter sich faßt.

Die Dinge liegren weit komplizierter, wie ursprünglich vermutet werden konnte. Viele Forderungen des Lebens laufen nebeneinander 
her, greifen vielseitig ineinander und ergänzen einander $z \mathfrak{u}$ e in e m Lebensganzen.

Es kann nur e in e Philosophie geben, welche das Leben, wie es ist, erklärt. Sind die Grundlagen einer Welt- und Lebensanschauung wahr, so müssen sich auch die Philosophien aller Zeiten, soweit sie lebenswahre Bestandteile in sich bergen, glatt in sie einfügen lassen.

Es müssen somit zunächst die Grundlagen der Welt- und Lebensanschatung festliegen, ehe jene Einzelwahrheiten als solche recht erkannt und an ihren Platz gerückt werden können; bis dahin können sie nur annähernd begrenzt und in ihrem Wert beurteilt werden.

Eine genügend fundierte Weltanschauung besitzen wir heute, aber es fehlt uns die Le be n san s cha u u $\mathrm{g}$, die dazu gehört, und die organisch aus ihr herauswachsen muß.

$\mathrm{Da}$ jede Lebensanschauung letzten Endes in eine Ethik ausmündet. die ihre Krönung bildet, so muß unser Streben darauf gerichtet sein, die ethische Lebenswahrheit $\mathrm{zu}$ finden, die eben auch nur aus dem Leben selbst abge le it et werden kann, nur in ihm selbst $s$ e in $\mathrm{e}$ He rk un ft haben kann.

Im Leben selbst muß sie also ihre nährenden Wurzeln schlagen, aus ihm ihre gesunde Kraft saugen, um sie dem sich entfaltenden h ö h e r e n Leben zuziführen. Nur dann kann das kulturelle Leben der Völker dauernden Bestand haben, nur dann ist es davor gesichert, fals $\mathrm{ch}$ e $\mathrm{n}$ Idealen: nachzujagen und in fạlscher Richtung. tiber das Ziel hinauszustreben, oder auch $\mathrm{zu}$ erschlaffen, $\mathrm{zu}$ entarten, in sich selbst zurückzusinken und zugrunde zu gehen.

Das Geschehen unserer Zeit drängt uns aber a ui Erkenntnis dieses höchsten Gutes hin, es verkörpertsich in der Entwicklung Deutschlands.

$*$

Die Ethik der allgemeinen Menschenliebe war, wie wir sagten. ein iundamentaler Bestandteil des Lebens und dieser dem Altruismus entspringende Teil versagte in der sich langsam vorbereitenden kritischen Situation der europäischen Menschheit. Den andern lebenswahren Bestandteil glaubten wir in der Entwicklungslehre finden $z u$ müssen. Aus der Lebensentwicklung muß sich der ne ue noch iehlende ethische Bestandteil ableiten lassen. Solange wir 
diesen noch nicht besitzen. greift die Lebensentwicklung auch bei uns Mensclien immer wieder auf den krassesten Exoismus, der dem biołogischen (des höheren Menschen unwürdigen) Daseinskampi zu Grunde liegt, zuriick.

So schen wir es zur Zeit des römischen Imperialismus, so auch in Nietzsches Gedankengängen und so schen wir es im gegenwärtigen Weltkrieg. Fr steht in engsten Zusammenhang mit dem unserm modurnen Leben fehlenden $\mathrm{Ha}$ uptbestandteil der Ethik. Er steht in engster Beziehung zu den größten Problemen der Menschheit. Sie kämpft den Daseinskampf in der gigantischten Form. die er jemals, solange die Erde steht, angenommen hat.

Die Welt erhält ein Beispiel von unierhörter Wucht; ein praktisches Beispiel von eindringlichster Kraft und Tiefe, von oben her$a b$, von höchster offizieller Seite gegeben, rückwirkend aui die gesamte Menschheit, sie vorbereitend auf das, was folgen muß:

Es spitzt sich das Weltgeschehen auf ein Prinzip zu! In Deutschland verkörpert sich das Entwicklungsprinzip und mit Deutschland ringt sich der Entwicklungsgedanke zur offiziellen Anerkennung seines Rechts durch. Sichentwickelndiirfen ist ein Recht, das an sich besteht, ist also ein Naturrecht, weil das Wesen alles Lebens auf Entwicklung begründet ist.

Mit dem Entwicklungskampf Deutschlands wird die gesamte Menschheit auf das Entwicklungsproblem: a uf En tw icklung als eine wirkende Macht in uns a ufmerksam, die ganz allgemein jedes Volk und jedén einzelnen Menschen betrifft. Die Entwicklungslehre als solche setztsich zur offiziellen Anerkennung durch. Sichentwickeln-dürien ist das erste Recht jedes Einzelmenschen und jedes Volkes. (Vergl. Grundgedanken nur neuen Ethik.)

$*$

Im Zentrum der christlichen Lebensanschauung steht die Nächstenliebe. ' Im Zentrum der Entwicklungslehre, die der monistischen Welt- und Lebensanschauung $z u$ Grunde liegt, steht der Daseinskampf, der im Egoismus wurzelt Altruismus und Egoismus stehen sich in zwei Weltanschaunungen gegenüber. Der Monismus lehnt den Altruismus aber keineswegs ab; im Gegenteil, er bemüht sich, ihn organisch einzugliedern, 
denn heute ist er nur erst lose angegliedert. Aber auch die christlichie Anschauung lehnt den Egoismus nicht grundsätzlich ab. Es gilt also eine Synthese zwischen Egoismus und Altruismus zu gewinnen und ihre giegenseitige organische $D$ u r ch dring ung herbeizufiihren, um eine einheitliche Lebensanschauung zu gewinnen. Es gilt. die in jedem qualifizierten harmonischen Menschen liegenden $\mathrm{Naturgrundlag}$ en der Seele, zu denen außer dem egoistischen und altruistischen Streben vor allem a it $\mathrm{h}$ id i $\mathrm{Seh} n \mathrm{such}$ t na ch einem vollkommeneren Leben gehört, die Sehnsucht aus den Unvollkommenheiten des Jetztzustandes herauszukommen, stark in den Vordergrund zu stellen, sinnvoll zi verstehen und den sehnsïchtigen Menschen den gangbaren Weg. zur Befreiung von der Not des Daseins zu weisen.

Egoismus, Altruismus und Sehnsucht nach größerer Vollkommenheit sind die drei Punkte, die wir lebenswahr zu verschmelzen haben.

Der Weg, wie wir die Unvollkommenheiten überwinden können, ist uns allein durch den deutschen Idealismus angezeigt und zwạr durch den praktischen Idealismus. Durch den praktischen Idealismus der Wirklichkeitsidealisten, der sachlich denkenden, real blickenden und aktiv schöpierischen Idealisten. können wir zu größerer Vollkommenheit gelangen. (Der praktische Idealismus steht keineswegs im Gegensatz zum philosophischen Idealismus, er geht vielmehr' glatt aus ihm hervor.)

Der Idealismus ist an Individuen gebunden. Das jdiealistisch und realistisch zugleich veranlagte Individuum ist nicht etwa ein zwitespältiges Wesen. sondern gerade ein harmonisches. Idealismus und Realismus ergänzen sich zı einem harmonischen Ganzen. (Vergl. Über das Wesen des Idealismus).

Da das realidealistische Einżelindividuum für seine Person und allein aui sich selbst gestellt nichts Großes verwirklichen kann, sondern dazı stets der Mitarbeit zahlreicher Hilfskräfte in Arbeitsteilung und durch Organisation einer größeren sozialen Gesamtheit bedarf; so ergibt sich daraus im Keime eine Synthese zwischen Individualismus und Sozialismus.

Den Wirklichkeitsidealisten kommt krait ihrer Fähigkeiten die höchste Leitung in a 11 e m, was Organisation beißt, zu, alle iibrigen nicht real-idiealistisch Veranlagten ordnen sich ein in seine Organi- 
sation; ein jeder hat an dem Platze zu stehen, der ihm nach seinen Fähigkeiten und Anlagen zusagt und zusteht.

Dic krassen Zustände im heutigen sozialen Gesellschaftsorganismus, wie sie zwischen Besitzenden und besitzlosen Arbeitern, zwischen Arbeitgebern und Arbeitnehmern bestehen, sind zurückzuführen auf den uns noch fehlenden ethischen Bestandteil, welcher aber aus dem Entwicklungsgedanken $\mathrm{zu}$ gewinnen ist und auf eine sittliche Disziplinierung"des egoistischen Sichauswirk ens hinausläuft, das, je weniger es sittlich gehemmt ist, einen umso krasseren Daseinskampi nach sich zieht. Alle Willensrichtungen bedürfen der gemeinsamen Orientierung an einem übergeordneten höheren Ganzen. Dieses Ganze ist die aufsteigende Lebensentialtung.

Das zukünftige Ganze liegt aber den in ne re $\mathrm{n}$ Willensrichtungen (den. Triebrichtungen in uns) bereits in der Anlage $z u$ Grunde, es wird bis jetzt nur noch nicht bewußt erkannt. Es ist das gleiche Prinzip, das die Ursache der gesellschaftlichen Harmonie bildet; denn trotz aller Streitigkeiten iällt der Gesellschaftsorganismus doch nie auseinander. Selbst aus den chaotischen Zuständien großer Revolutionen haben sich, wenn auch nie ideale Zustände, so doch immer wieder relativ harmonische Verhältnisse und meistens auch bessere wie zuvor herausgestaltet.

Die Summe der zentrifugalen und zentripetalen Willensrichtungen trägt die Harmonie bereits in sich. Wir können aber die uns von Natur gesteckten Ziele erst bewußt anstreben, wenn wir sie erkannt haben, und damit würden wir zugleich große, alle Menschen vereinende Gedanken gewinnen.

Gelingt es uns, die ethische Lebenswahrheit" $z u$ finden, so wird es uns klar werden, wie die Streitfragen zwischen Individualismus und Sozialismus, zwischen Egoismus und Altruismus, zwischen Realismus und Idealismus, Nationalismus und Universalismus, Monismus und Dualismus, Wissenschaft und Religion in der Tiefe alle miteinander zusammen hängen.

Auf der ethischen Lebenswahrheit baut sich das Rechtsgebäude der sozialen Gesellschaft auf, die Wahrheit dient den Rechtsanschanungen als Grundlage. Gesellschaft und Mienschheit erhalten also ein gemeinsames Fundament. 
Entsprechend der ungeheuren Größe der Katastrophe der Menschen ist auch die Bedeutung der aus ihr hervorgehenden Wahrheiten für sie. Noch nie, solange unser Planet besteht, hat sich Ähnliches auf ihm zugetragen, und demgemäß ist auch derr geistige Aufschwung, der die Menschlieit in der Tiefe aufrütteln wird.

Die Bedeutung der gegenwärtigen Zeit ist also darin zu erblicken, daB das Weltgeschehen auf eine Verschmelzung der Grundgedanken des dogmenfreien Christentums mit den Grundgedanken der naturwissenschaftlichen Forschung: der Entwicklungslehre hinausdrängt, oder mit anderen Worten: Aus den gewaltigen Zeitereignissen, wird eine Verbrüderung zwischen Wissenschaft und Religion hervorgehen, die Hand in Hand dem gemeinsamen, natürlich-göttlichen Ziele zustreben, ein vollkommeneres Leben herbeizuführen.

Wir stehen vor dieser entscheidenden Wende und werden sie erleben. 